\title{
Assessment of a single monomorphic ventricular ectopy from the right ventricular outflow tract in standard and high resolution electrocardiogram
}

\author{
Dariusz Kozłowski ${ }^{1}$, Adam Kosiński², Alicja Dąbrowska-Kugacka ${ }^{1}$, Ewa Lewicka-Nowak', \\ Maria Dudziak ${ }^{3}$, Marek Grzybiak², Grzegorz Raczak ${ }^{1}$
}

1Department of Cardiology and Electrotherapy, Medical University of Gdansk, Poland
2Department of Clinical Anatomy, Medical University of Gdansk, Poland
${ }^{3}$ Noninvasive Cardiac Diagnostic Department, Medical University of Gdansk, Poland

Submitted: 1 December 2008

Accepted: 4 August 2010

Arch Med Sci 2010; 6, 5: 713-718

DOI: 10.5114 /aoms.2010.17086

Copyright (c) 2010 Termedia \& Banach

\section{Abstract}

Introduction: High-resolution electrocardiography (ECG-CREM) is a method based on digital electrocardiography. In order to facilitate the interpretation of the Crem records the technique of vectorcardiography was used. In comparison the origin of the ventricular premature complexes (VPCs) could be estimated based on a standard 12-lead electrocardiogram. The aim of the study was to assess the point of origin of the VPCS in ECG-CREM and correlate it with standard electrocardiography (ECG-Stand).

Material and methods: Our study included 26 patients (16 females, 10 males), aged 51-83 years (avg. 58.1 \pm 12.3 ), who presented with recurrent, during at least 6 months' observation, VPCs. The point of origin of VPCs was compared in both methods.

Results: The performed analysis of collected ECG-Stand records revealed the presence of arrhythmogenic focal points in six different locations $(1,3,5,7,8,9)$. However, we did not affirm their presence in points $2,4,6$. They were most commonly located in RVOT zones 8 (30.7\%), 9 (23.0\%), 5 (23.0\%), and most seldom in zones 1, 3, 7 (7.6\% each). In the simultaneous record of ECG-CREM with a single VPC it was confirmed that the FPb zone was activated the most frequently (40.0\%); the next in relation to frequency were SD and ST $(20.0 \%)$. Less frequent VPCs have their origin in Crem zones SP, FPa and SB (6.6\%). Conclusions: Electrocardiogram of high signal resolution (ECG-CREM) might be useful in recognition of the origin of ventricular premature complexes from RVOT.

Key words: ventricular ectopy, RVOT, standard ECG, high-resolution electrocardiography.

\section{Introduction}

Idiopathic ventricular tachycardia (VT) with ventricular premature complexes (VPCs) in patients without organic heart disease is the most benign form of ventricular arrhythmia [1]. Risk of serious cardiovascular incidents including sudden cardiac death is comparatively low in this group of patients [2]. Unfortunately, the majority of them have uncomfortable symptoms of a beating heart, e.g. palpitations. Major idiopathic ventricular arrhythmias are: tachycardia type I (repetitive monomorphic VT) as well as type II (idiopathic tachycardia from left or right ventricle) [3]. Morphologically, the origin of right ventricular arrhythmias could be the

\author{
Corresponding author: \\ Dariusz Kozłowski, MD, PhD \\ Department of Cardiology and \\ Electrotherapy \\ Medical University of Gdansk \\ ul. Dębinki 7 \\ 80-211 Gdansk, Poland \\ Phone: +48 583493910 \\ E-mail: dkozl@gumed.edu.pl
}


right ventricle outflow tract (RVOT) or the right ventricle inflow tract (RVIT). Most frequently VPCS are generated from the RVOT. In the majority of cases the site of origin is localized from a narrow segment in the RVOT, called the anterior septum, near the pulmonary valve. Occasionally, VT originates from the free wall and the posterior septal aspect of the RVOT [4].

On standard 12-lead electrocardiogram VPCs originating from the right outflow tract manifest left bundle branch block morphology and inferior frontal plane axis ( $\angle B B B$ with the axis $90^{\circ}$, transitional zone in $V_{3}-V_{4}$ and high $R$ in II, III, aVF) [4]. Identification of septal and free wall sites based on ECG however is very difficult. On the other hand, extrasystole generated within the RVIT manifest in low R in II, III, $\mathrm{aVF}$, and an early transitional zone in $\mathrm{V}_{2}$. Based on those criteria electrocardiographic localization is still difficult due to the presence of complicated anatomical relations [5]. Exact localization was initially described by Marchlinski et al. [3]. The authors divided the outflow of the right ventricle into two separate planes. In the frontal plane the appearance of $\mathrm{R}$ in lead I localized arrhythmia in the posterior part of the RVOT (close to the tricuspid valve). However, QS or Qr complexes reflect the position in the anterior part of the RVOT. The occurrence on ECG of qR complexes in lead I was most characteristic for the central part of the RVOT. In the horizontal plane appearance of $R$ greater than $\mathrm{QS}$ in $\mathrm{V}_{3}$ takes a main role. When $\mathrm{R}$ was higher than $S(R>S)$ in lead $V_{3}$, origin of the arrhythmia in the central and bottom part of the RVOT was excluded. However, passage from $r<S$ to $R>s$ in precordial leads $V_{4}-V_{6}$ shows the position in the bottom part of the RVOT. The differential diagnosis of idiopathic RVOT ventricular tachycardias on 12-lead ECG still remains very difficult.

The new method using electrocardiography of high signal resolution (ECG-CREM) is based on digitally recorded 12 -lead ECG and afterwards digital processing of the recorded signal. Surface ECG leads are applied in the standard manner and the electrocardiogram is recorded onto Frank's orthogonal plane. The signal obtained during recording is analysed in terms of electric activity regarding many different zones of the heart's ventricle. Six sites were differentiated based on the ECG-CREM: SP (the anterior zone), ST (the posterior zone), PPG (the septal zone) and SD (the inferior zone). The use of ECG-CREM seemed to be more precise in the analysis of the front of activation of premature beats ventricular in origin $[6,7]$. Therefore we decided to use an alternative diagnostic method to assess ventricular paroxysmal contractions on high resolution ECG and correlate the results with the results obtained from standard ECG [8].

\section{Material and methods}

Our study included 26 patients (16 females, 10 males), aged 51-83 years (avg. 58.1 \pm 12.3 ), who presented with recurrent, for at least 6 months' observation, VPCs. The study population consisted of patients without structural heart disease. Holter monitoring and ECG-CREM and standard electrocardiography (ECG-Stand) were preceded by detailed history collection and thorough physical examination. Additional examinations also took place, especially echocardiography and exercise test. Differential diagnosis was made to exclude other (e.g. structural) causes of the occurrence of VPCS (ischaemic heart disease, cardiomyopathy, heart failure, electrolyte disorders, drug overdose, QTC assessment).

Examinations were carried out in the Syncope Diagnostic Unit of the Department of Cardiology and Electrotherapy of the Medical University of Gdansk, according to the standard protocol. Examinations were conducted at a fixed time of day, in the morning. Drugs that might affect the circulation had been withdrawn respecting a 3-fold half-life period before the examination. During the examination the test room was equipped with a defibrillator and resuscitation drugs. In each patient a standard 12-lead electrocardiogram and high resolution electrocardiogram were performed preceded by a 10-minute resting period in a supine position. Our goal was to localize the point of origin of ventricular premature beats. All patients at the time of examination presented spontaneous VPC activity. Localizations of arrhythmia origin as a result of performed investigations of ECG-Stand and ECG-CREM were correlated.

The signal obtained during spontaneous VPC recordings were analysed in terms of electrical activity regarding many fragments of the heart's myocardium. Six sites were differentiated based on the ECG-CREM: SP (the anterior zone), ST (the posterior zone), PPG (the septal zone) and SD (the inferior zone). Additionally the ECG-CREM was divided into three paraseptal groups: FPb (the central-posterior zone), FPa (the central-anterior zone) and LOT (the posterior-inferior-lateral zone). In comparison to standard 12-lead ECG QRS morphology was analysed in the three standard and three augmented limb leads and the precordial leads. The nine sites were divided into three groups: anterior region of RVOT, intermediate zone of RVOT and posterior zone of RVOT.

\section{Results}

In all cases (26), monomorphic ventricular premature beats were recorded in amounts ranging from 12.430 to 39.860 per 24-hour period (avg. $22.834 \pm 926$ ). In 12-lead routine ECG in all 
cases single monomorphic ventricular premature beats were observed with broad ( $\geq 120 \mathrm{~ms}$ ) QRS complexes, left bundle branch morphology (LBBBnegative deflections in leads $V_{1}-V_{2}$ and positive forces in $V_{5}-V_{6}$ ), inferior frontal plane axis (monophasic R waves in leads II, III, aVF) and QS pattern in leads $\mathrm{QVR}$ and $\mathrm{aVL}$. All ventricular paroxysmal contractions fulfilled the criteria of VPCs' origin from the right ventricular outflow tract (RVOT). Because of the constant coupling interval in relation to the normal QRS complexes premature beats were described as extrasystolic or parasystolic (avg. $420 \pm 34 \mathrm{~ms}$ ). In all cases evidence for the existence of a trigger zone was found. The location of it was established according to Marchliński's criteria [3]. This classification divided RVOT into two planes: frontal and horizontal. The frontal plane consists of three zones located in the right ventricle outflow tract (anterior numbers: 3, 6, 9; as well as posterior numbers 1, 4, 7, and also intermediate: $2,5,8)$. Similarly, three zones of the horizontal plane were distinguished (superior numbers: 1, 2, 3; inferior numbers: 7, 8, 9 and central numbers: 4, 5, 6). This classification is based on the existence in lead I of complexes R/Rs (posterior or intermediate position), qR (intermediate or anterior position), QS/QR (anterior position). Presence of $R$ in the $\mathrm{aVL}$ lead favours a posterior or intermediate position, and lack of $R$ in this lead favours a central or anterior position. In addition to these criteria a transitional zone of $R$ into $S$ in lead $V_{3}$ was also counted. If this occurred in $V_{3}$ ( $V_{3}$-yes) this was called an early transitory zone $(R>S)$ and this favoured the centralsuperior position (numbers: $1,2,3,4,7$ ). In other cases $\left(V_{3}\right.$-no) the central-inferior position was observed (numbers: 5, 6, 8, 9). Based on the results of our study we established that the trigger zone might be located in six different positions $(1,3,5$, $7,8,9)$; however we have not observed it in other positions $(2,4,6)$.

Based on our study in position number 1 (superior-posterior region) arrhythmia occurred in 2 patients (ECG-Stand: I-R + aVL $-\mathrm{QS}+\mathrm{V}_{3}$-yes) $7.6 \%$. In position number 3 (superior-anterior region) VPCS appeared in 2 patients (ECG-Stand: I - QS + $\mathrm{aVL}-\mathrm{QS}+\mathrm{V}_{3}$-yes) $-7.6 \%$. In position number 5 (central-intermediate region) arrhythmia occurred in 6 patients (ECG-Stand: I - Rs + aVL $-q S+V_{3}-n o$ ) $-23.0 \%$. In Figure 1A, the 12-lead ECG is presented with the origin of VPCs from region number 5 $(\hat{\jmath}, 22$-year-old). In position number 7 (inferiorposterior region) VPCs were confirmed in 2 patients (ECG-Stand: I - RS + aVL - Rs + $\mathrm{V}_{3}$-yes) $-7.6 \%$. In position number 8 (inferior-intermediate region) ECG was characterized by I $-R+a V L-R+V_{3}$-no and it appeared in 8 patients (30.7\%). Meanwhile, in position number 9 (inferior-anterior region) ECG had the features: I $-\mathrm{QS} / \mathrm{QR}+\mathrm{aVL}-\mathrm{QS}+\mathrm{V}_{3}$-no and such a record was observed in 6 cases (23.0\%). We were not able to find any correlation between the position of the trigger zone and patients' sex or age. The ECG-CREM was executed twice: the first during normal sinus rhythm and the second during spontaneous ventricular extrasystoles. Unfortunately, only 15 of 26 records were completed and enabled further processing and assessment. The result analysis during sinus rhythm without VPCS revealed the highest values of electrical activity, normalized as well as standardized, in the inferior wall zone (SD). Electrical activity ranged in this zone from $15 \mathrm{mV}^{2}$ to $309 \mathrm{mV}^{2}$ (average 160.4 $\pm 74.8 \mathrm{mV}^{2}$ ). The next part of the heart muscle with high electric activity was the posterior wall zone (ST). The mean value of relative electrical activity was $60.2 \pm 39.8 \mathrm{mV}^{2}$ (range: 8 to $108 \mathrm{mV}^{2}$ ). The next highest zone with high electrical activity was the posterior-inferior-lateral zone (LOT) with a range of values from 3 to $106 \mathrm{mV}^{2}$ and average $32.6 \pm 41.7 \mathrm{mV}^{2}$. The mean value of electrical activity, normalized as well as standardized, was lowest in the anterior-septal zone (PPG) and highest in the posterior zone (ST). The difference between those two zones was statistically non-significant $(p>0.05)$. Data are shown in Table I.

During VPCs the analysis of results showed the highest values in the central-posterior zone (FPb). Relative activity ranged in this zone from 5 to $347 \mathrm{mV}^{2}$ (average $87.8 \pm 101.3$ ). This zone was activated most frequently (6 cases, $40.0 \%$ ). In Figure $1 B$ the ECG-CREM is presented with the origin of VPCs from the zone FPb (§, 22-year-old). The next fragment of the heart muscle with high electric activity was the posterior zone (ST). The range of values is from 8 to $108 \mathrm{mV}^{2}$ (average 60.2 $\pm 39.8)$. This fragment was activated during VPCs in 3 cases (20.0\%). The same frequency of activation appeared in the inferior zone (SD) - 3 cases (20.0\%). In one patient $(6.6 \%)$ changes in electrical activity of VPCs in the form of central-anterior zone (FPa) activation were observed. The other fragments did not present amplified activity on ECG-CREM. Precise data are shown in Figure 2.

The comparison of ECG-Stand and ECG-CREM unfortunately was very difficult. In standard ECG we are able to assess more or less 9 zones (numbers 1-9), in high resolution ECG 8 zones (abbreviations: FPb, FPa, SD, ST, SP, SB, LOT, PPG). Appropriate regions on standard ECG assess exactly RVOT, while using high resolution ECG gives the direction of the strongest activation. In both methods we have noticed zones not activated by VPCS. On ECG-Stand they were regions 2, 4, 6 and on ECG-CREM they were LOT, PPG. On ECG-CREM the $\mathrm{FPb}$ zone was the most frequently activated (40.0\%), on ECG-Stand the most frequently activated was the inferior-intermediate (number 8) 
region (30.7\% of cases). The next zones less activated were the inferior wall (SD) on ECG-CREM and inferior-anterior zone (No. 9) or centralintermediate zone (No. 5) in ECG-Stand. The former was observed in $20 \%$ of patients, and the latter in $23.0 \%$. Less frequent VPCs have their origin in regions: no. 1 (superior-posterior), no. 3 (superioranterior) and no. 7 (inferior-posterior) - 7.6\% each. In ECG-CREM those zones were as follows: SP

\section{A}

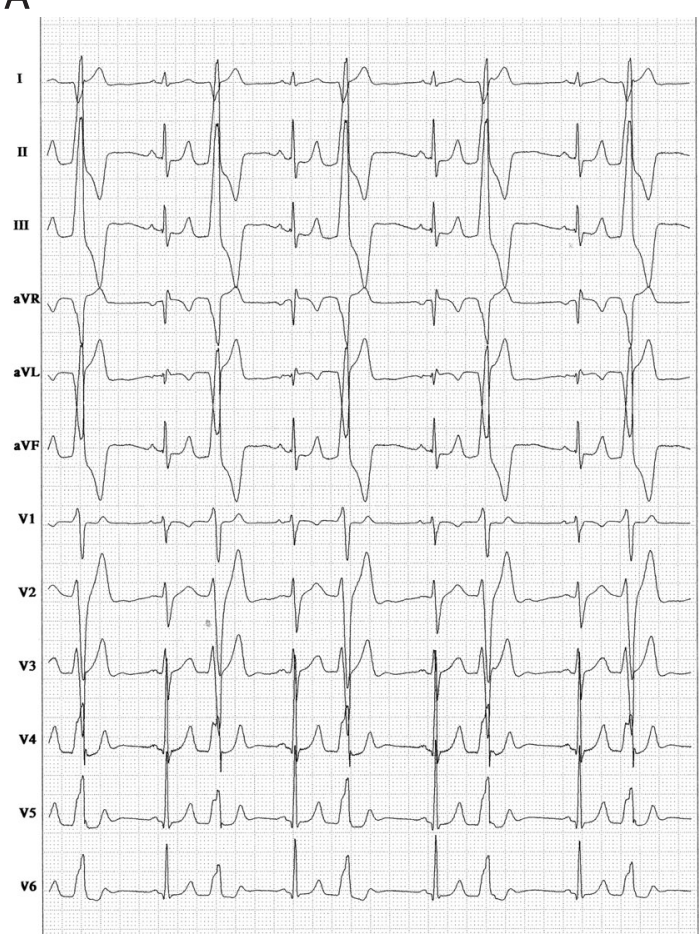

(anterior zone), FPa (central-anterior zone) and SB (lateral zone) $-6.6 \%$ each. Furthermore, on ECGCREM there were ST and FPa localization, which do not correspond to any location described by ECGStand (13.3\%).

\section{Discussion}

Tachycardia from the right ventricle outflow tract is a great challenge for cardiologists. Most of them are moderate in course and do not correspond to 10-year mortality [9]. Patients with the diagnosis of idiopathic VT or VPCs are completely healthy. This concerns especially muscle, where echocardiography ultrasonography investigation is without any pathology. However, some patients have minimal changes regarding the structure of the heart based on advanced tests such as NMR, SPECT, PET [10, 11].

In available publications we are able to find lots of information regarding characteristic ECG signs of tachycardia initiated in the RVOT [4-6, 12]. Unfortunately, our findings on ECG records do not correspond to those established in the literature during ablation procedures. The most probable explanation of this phenomenon is the method of collecting data during pace mapping in electrophysiological study (EPS). The EPS method is based on the presence of QRS complexes artificially generated in different projections, and so obtained records are compared to those from a standard 12-lead ECG. In this technique the RVOT is normally divided into anterior, posterior, superior and inferior regions and diagnosis is based on the deflection of the QRS complex in limb leads (mainly $\mathrm{I}$ and $\mathrm{aVL}$ ) as well as precordial $\left(\mathrm{V}_{3}\right)[14,15]$. Unfortunately, mistakes can occur here as well,

B
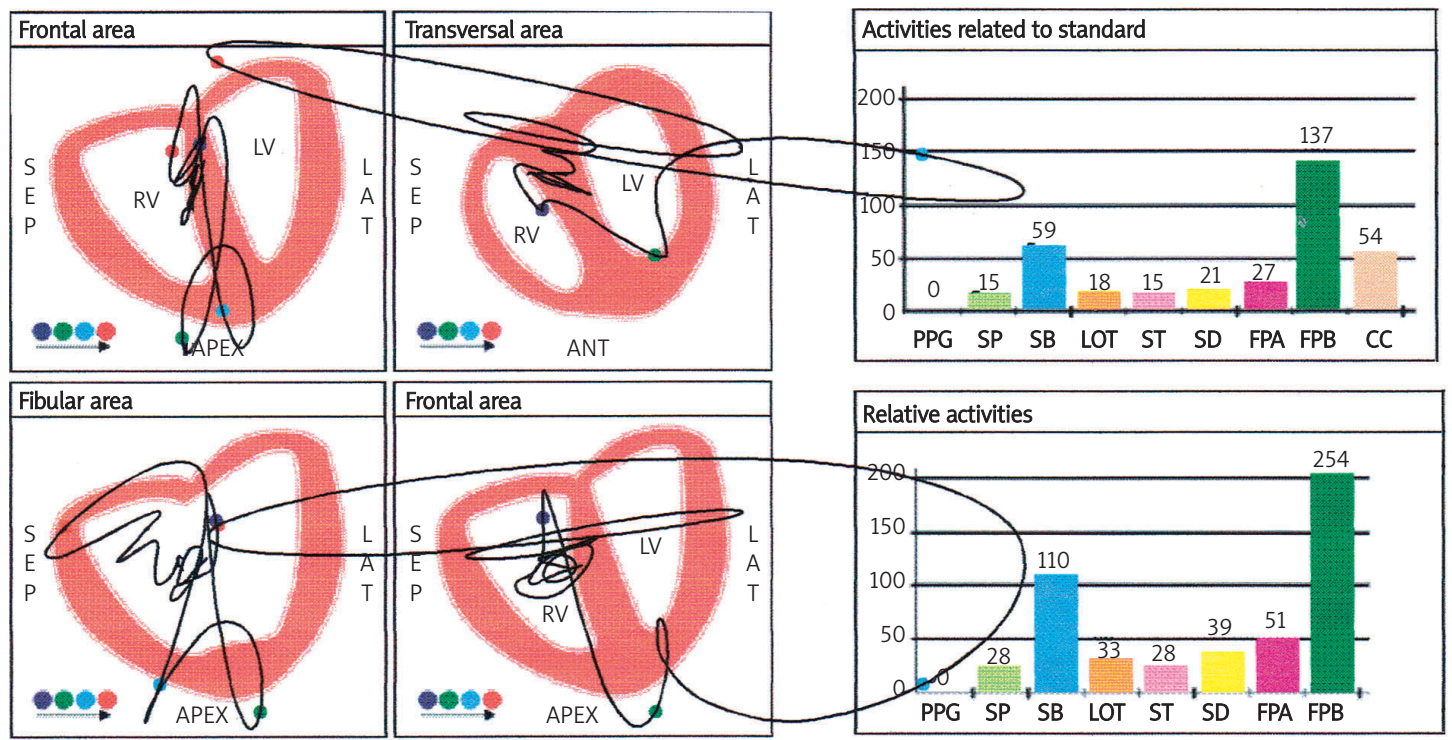

Figure 1. Location of trigger zone on ECG-Stand and ECG-CREM in patient W.L., ô, 22-year-old. A-12-lead ECG is presented with the origin of VPCS from region number 5. B - ECG-CREM is presented with the origin of VPCs from zone $\mathrm{FPb}$ and $\mathrm{SB}$ 
Table I. Average level of electrical activity depending on region of heart on ECG-CREM in the presence of single VPC

\begin{tabular}{|lccccccccl|}
\hline & \multicolumn{10}{c|}{ Relative activity $(\mathrm{mV}$ ) $)$ in relation to the presence of VPCS } \\
\hline & PPG & SP & SB & LOT & ST & SD & FPa & FPb & Main location on ECG-CREM \\
\hline EF & 4 & 3 & 7 & 74 & 16 & 105 & 45 & 130 & CENTRAL-posterior zone $(F P b)$ \\
\hline SS & 7 & 22 & 174 & 108 & 24 & 55 & 13 & 189 & CENTRAL-posterior zone (FPb) \\
\hline FK & 25 & 17 & 57 & 32 & 14 & 23 & 46 & 60 & CENTRAL-posterior zone (FPb) \\
\hline EJ & 7 & 6 & 11 & 79 & 106 & 89 & 10 & 17 & Posterior zone (ST) \\
\hline Bt & 91 & 114 & 17 & 8 & 3 & 27 & 18 & 74 & Anterior zone (SP) \\
\hline JJ & 7 & 6 & 57 & 152 & 96 & 228 & 10 & 9 & INFERIOR zone (SD) \\
\hline ZK & 8 & 14 & 61 & 160 & 162 & 210 & 10 & 11 & INFERIOR zone (SD) \\
\hline GM & 4 & 3 & 11 & 244 & 446 & 236 & 5 & 21 & Posterior zone (ST) \\
\hline PM & 6 & 6 & 24 & 23 & 15 & 15 & 10 & 5 & Lateral zone (SB) \\
\hline MM & 7 & 8 & 6 & 268 & 510 & 309 & 10 & 28 & Posterior zone (ST) \\
\hline FJ & 54 & 71 & 48 & 80 & 18 & 134 & 59 & 238 & CENTRAL-posterior zone (FPb) \\
\hline TW & 7 & 6 & 80 & 84 & 26 & 116 & 10 & 347 & CENTRAL-posterior zone (FPb) \\
\hline ZE & 35 & 21 & 21 & 46 & 43 & 64 & 28 & 14 & INFERIOR zone (SD) \\
\hline WL & 4 & 3 & 7 & 74 & 116 & 105 & 45 & 130 & CENTRAL-posterior zone (FPb) \\
\hline GJ & 38 & 73 & 95 & 70 & 37 & 80 & 173 & 45 & CENTRAL-anterior zone (FPa) \\
\hline & 20.27 & 24.9 & 45.1 & 100.1333 & 108.8 & 119.7333 & 32.8 & 87.866667 & Average (avg.) \\
\hline & 24.96 & 33.5 & 45.9 & 75.88042 & 157.4544 & 88.07746 & 42.54443 & 101.34937 & Standard deviation (SD) \\
\hline
\end{tabular}

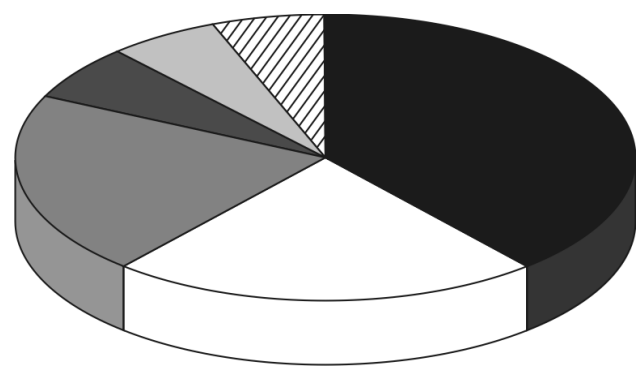

- [FPb]-CENTRAL-posterior (6 patients - 40\%)

$\square$ [SD]-INFERIOR (3 patients - 20\%)

口 [ST]-POSTERIOR (3 patients - 20\%)

— [FPa]-CENTRAL-anterior (1 patients - 6.6\%)

[SP]-ANTERIOR (1 patients $-6.6 \%)$

[SB]-LATERAL (1 patients - 6.6\%)

Figure 2. Location of trigger zone in ECG-CREM. Upper case marks frontal plane, e.g. CENTRAL, lower case marks horizontal plane, e.g. posterior

probably because of variability in patients' hearts' RVOT anatomy. According to available knowledge, the RVOT has a very complicated anatomical structure $[16,17]$. The RVOT region is defined superiorly by the pulmonary valve and inferiorly by the superior margin of the right ventricle inflow tract - e.g. the tricuspid valve. The interventricular septum and right ventricle free wall constitute the medial and lateral aspects respectively. According to the Japanese anatomist Adachi, RVOT structure is an individual structure in the heart and from the clinical point of view is characterized by great

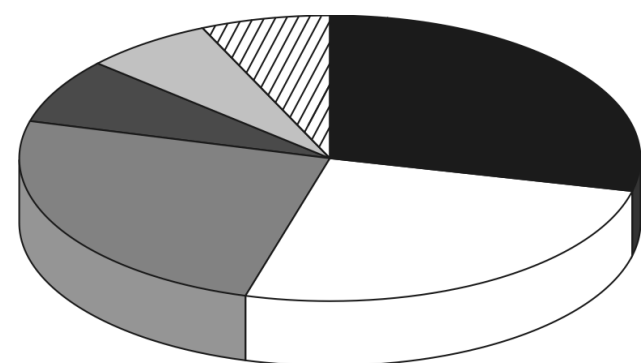

[8]-INFERIOR-intermediate (8 patients - 30.7\%)

$\square$ [5]-CENTRAL-intermediate (6 patients - 23\%)

$\square$ [9]-INFERIOR-anterior (6 patients - 23\%)

口 [7]-INFERIOR-posterior (2 patients - 7.6\%)

$\square$ [3]-SUPERIOR-anterior (2 patients $-7.6 \%$ )

[1]-SUPERIOR-posterior (2 patients - 7.6\%)

Figure 3. Location of VPC's origin on ECG-Stand. Upper case marks frontal plane, e.g. CENTRAL, lower case marks horizontal plane, e.g. posterior

variation. This anatomical variability makes difficult proper location of the trigger zone in connection with standard ECG.

Therefore one of the new methods based on electric activity of individual parts of the heart muscle is the electrocardiogram of high signal resolution. There are no impact analyses concerning ECG-CREM in available publications, but it seems to be quite a promising diagnostic method. Some initial tests have revealed a correlation between ECG-CREM and heart ultrasonography in patients after myocardial infarction. Unfortunately, according 
to Lepska, such a correlation does not exist [7]. However, other authors, based on their initial research concerning patients with incessant premature ventricular complexes, reported statistically significant differences in electrical activity occurring only in FPa (decline in activity, $p=0.002$ ) and the lateral wall SB (decline in activity, $p=0.05$ ). Unfortunately, because of the small number of examined patients the results were not sufficient to formulate a hypothesis. Initially analysed electrical activity in heart muscle zones during sinus rhythm reveals no statistical differences.

In our research we established that the most commonly activated fragment during VPCs was the central (frontal plane)-posterior (horizontal plane) zone on ECG-CREM and the inferior (frontal plane)intermediate (horizontal plane) zone on ECG-Stand. The next, regarding frequency of occurrence, were the inferior zone (frontal plane) on ECG-CREM and the central (frontal plane)-intermediate (horizontal plane) zone on ECG-Stand. According to this we observe an approximate correlation between those two diagnostic methods, in which most frequently the VPC'S origin was in the central region of the RVOT. But the group of examined patients was not large enough to detect any significant correlation. The ECG-CREM method requires further study with a larger patient group. Additionally, the ECG-CREM algorithm should be modified focusing on location of VPCs. The most appropriate research should be based on comparison of high resolution electrocardiography with EPS procedure.

In conclusions, (1) electrocardiogram of ECG-CREM might be useful in recognition of the origin of ventricular premature complexes from the RVOT. (2) It was observed that on the ECG-Stand the position of the trigger zone could be well located within the RVOT; however, it is difficult to correlate it with ECG-CREM. (3) Precise location of the origin of the arrhythmia requires new assessment algorithms based on a large number of patients with simultaneous mapping during an effective ablation procedure.

\section{References}

1. Ouyang F, Fotuhi P, Ho SY, et al. Repetitive monomorphic ventricular tachycardia originated from the aortic sinus cup. J Am Coll Cardiol 2002; 39: 500-8.

2. Kochanska A, Zarzycka B, Świątecka G, et al. Quality of life In patients with an implantable cardioverterdefibrillator - the significance of clinical factors. Arch Med Sci 2008; 4: 409-16.

3. Jadonath RL, Schwartzman DS, Preminger MW, et al. Utility of the 12-lead electrocardiogram in localizing the origin of right ventricular outflow tract tachycardia. Am Heart J 1995; 130: 1107-13.

4. Tanner H, Wolber T, Schwick N, Fuhrer J, Delacretaz E. Electrocardiographic pattern as a guide for management and radiofrequency ablation of idiopathic ventricular tachycardia. Cardiology 2005; 103: 30-6.

5. Yoshida Y, Hirai M, Murakami Y, et al. Localization of precise origin of idiopathic ventricular tachycardia from the right ventricular outflow tract by a 12-lead ECG: a study of pace mapping using a multielectrode "basket" catheter. Pacing Clin Electrophysiol 1999; 22: 1760-8.

6. Yang Y, Saenz LC, Varosy PD, et al. Using the initial vector from surface electrocardiogram to distinguish the site of outflow tract tachycardia. Pacing Clin Electrophysiol 2007; 30: 891-8.

7. Łepska L, Romanowicz P, Świątek-Brzeziński K, et al. Korelacja wektokardiografii wysokiej częstotliwości (CREM) oraz scyntygrafii perfuzyjnej serca (SPECT) $W$ identyfikacji niedokrwiennych zmian mięśnia sercowego [Polish]. Folia Cardiol 2006; 12 (Supl. A): 12.

8. Haqqani HM, Morton JB, Kalman JM. Using 12-lead ECG to localize the origin of atrial and ventricular tachycardias: part 2 - ventricular tachycardia. J Cardiovasc Electrophysiol 2009; 20: 825-32.

9. Ainsworth CD, Skanes AC, Klein GJ, Gula LJ, Yee R, Krahn AD. Differentiating arrhythmogenic right ventricular cardiomyopathy from right ventricular outflow tract ventricular tachycardia using multilead QRS duration and axis. Heart Rhythm 2006; 3: 416-23.

10. Wadghiri YZ, Schneider AE, Gray EN, et al. Contrastenhanced MRI of right ventricular abnormalities in Cx43 mutant mouse embryos. NMR Biomed 2007; 20: 366-74.

11. Bonatto RC, Fioretto JR, Okoshi K, et al. Percentile curves of normal values of echocardiographic measurements in normal children from the central-southern region of the State of Sao Paulo, Brazil. Arq Bras Cardiol 2006; 87: 711-21.

12. Goncalves LF, Espinoza J, Romero R, et al. Fourdimensional fetal echocardiography with spatiotemporal image correlation (STIC): a systematic study of standard cardiac views assessed by different observers. J Matern Fetal Neonatal Med 2005; 17: 323-31.

13. Dixit S, Gerstenfeld EP, Callans DJ, Marchlinski FE. Electrocardiographic patterns of superior right ventricular outflow tract tachycardias: distinguishing septal and freewall sites of origin. J Cardiovasc Electrophysiol 2003; 14: 1-7.

14 Yamauchi Y, Aonuma K, Takahashi A, et al. Electrocardiographic characteristics of repetitive monomorphic right ventricular tachycardia originating near the Hisbundle. J Cardiovasc Electrophysiol 2005; 16: 1041-8.

15. Jadonath RL, Schwartzman DS, Preminger MW, Gottlieb CD, Marchlinski FE. Utility of the 12-lead electrocardiogram in localizing the origin of right ventricular outflow tract tachycardia. Am Heart J 1995; 130: 1107-13.

16. Kozłowski D, Kozłowska M. Anatomia kliniczna serca dla elektrofizjologów [Polish]. Lubiński A, Trusz-Gluza M, Walczak F, editors. Podręcznik elektrofizjologii klinicznej. Via Medica 2007, 1-14.

17. Vaseghi M, Cesario DA, Mahajan A, et al. Catheter ablation of right ventricular outflow tract tachycardia: value of defining coronary anatomy. J Cardiovasc Electrophysiol 2006; 17: 632-7.

18. Kozłowski D, Olszewski R, Dąbrowska-Kugacka A, et al. Ekstrasystolia z drogi odpływu prawej komory w standardowym elektrokardiogramie [Polish]. Folia Cardiol Exerpta 2008; 3: 578-85. 